\title{
Frequent Copy Gain of the MET Gene in Hypopharyngeal and Laryngeal Cancer in the Japanese Population
}

\author{
Ken Akashi1,2, Yasuhiro Ebihara, 1,2,3, Go Omura22, Yuki Saito², Masafumi Yoshida2, \\ Mizuo Ando ${ }^{2}$, Takahiro Asakage ${ }^{2,4}$, Tatsuya Yamasoba' ${ }^{2}$, Yoshinori Murakami1 \\ ${ }^{1}$ Division of Molecular Pathology, Institute of Medical Science, The University of Tokyo, Tokyo, Japan \\ ${ }^{2}$ Department of Otolaryngology-Head and Neck Surgery, The University of Tokyo, Tokyo, Japan \\ ${ }^{3}$ Department of Head and Neck Surgery, Saitama Medical University International Medical Center, Saitama, \\ Japan \\ ${ }^{4}$ Department of Head and Neck Surgery, Tokyo Medical and Dental Graduate School of Medicine, Tokyo, Japan \\ Email: ymurakam@ims.u-tokyo.ac.jp
}

Received 19 October 2015; accepted 24 November 2015; published 27 November 2015

Copyright (C) 2015 by authors and Scientific Research Publishing Inc.

This work is licensed under the Creative Commons Attribution International License (CC BY). http://creativecommons.org/licenses/by/4.0/

(c) (i) Open Access

\begin{abstract}
Molecular targeting therapy to specific genetic alterations has not been established in head and neck squamous cell carcinoma (HNSCC) except for cetuximab treatment. To characterize alterations of actionable oncogenes in HNSCC, we examined the gain of copy and mutation of the MET gene in 54 Japanese HNSCC. Copy gain of the MET was analyzed by droplet digital PCR (ddPCR) and quantitative real time PCR (qPCR) using 2 distinct fragments of the gene, and mutation was examined in exons 14 - 19 of MET by Sanger sequencing. Both ddPCR and qPCR showed significantly correlated results in copy number at two distinct fragments of the MET gene $(R=0.96$ and $R=$ 0.78), although ddPCR gave more significant and sensitive results. Copy gain of the MET was detected in 10 of $54(19 \%)$ HNSCCs and more frequently observed in tumors of the hypopharynx (4 of $12 ; 33 \%)$ or larynx $(5$ of $13 ; 38 \%)$ than those of the oral cavity $(1 \mathrm{of} 21 ; 4 \%)$ or oropharynx $(0$ of 8; 0\%), suggesting the existence of site-specific features in the oncogenic mechanisms of HNSCCs. Copy gain of the MET was also observed preferentially in older patients, although no correlation in other parameters, including clinical stages and overall or recurrence-free survival, was observed. On the other hand, of the two HNSCCs in which nucleotide substitution was detected, one was R1040Q in exon 15 with unknown function, and the other was a silent mutation in exon16. These results suggest that copy gain of the MET can provide an indicator for treatment with tyrosine kinase inhibitors for MET in a subset of hypopharyngeal or laryngeal cancer.
\end{abstract}

\section{Keywords}

Head and Neck Squamous Cell Carcinoma, MET, Copy Gain, Mutation, Molecular 


\section{Targeting Therapy}

\section{Introduction}

Head and neck squamous cell carcinoma (HNSCC) is the sixth most common cancer in the world and more than 300,000 people die of this disease every year [1]. More than half of HNSCC cases are diagnosed as advanced stage at the first examination. The 5-year survival rate of HNSCC patients is only about $40 \%$ and has not been improved essentially in the past three decades in spite of numerous trials in treatment, such as extended surgery, new chemotherapeutic agents and multimodal therapy [2].

Recently, on the basis of the advances in molecular understanding of each cancer, various molecular targeted therapies inhibiting specific addictive pathways in individual cancer cells have been established and have improved the therapeutic effect and prognosis of patients in several malignancies. Representative molecular targeting drugs for specific cancers as well as companion markers that have been established include imatinib for chronic myelocytic leukemia with bcr-abl fusion, gefitinib or crizotinib for non-small cell carcinoma (NSCLC) with the EGFR mutation or the $A L K$ fusion, respectively, and trastuzumab for breast cancer with overexpression of HER2. Cetuximab, a monoclonal antibody against epidermal growth factor receptor (EGFR), was shown to have an additional effect on locoregionally advanced HNSCC when combined with radiotherapy and to improve survival in recurrent or metastatic HNSCC when combined with conventional chemotherapy [3] [4]. Cetuximab was, therefore, approved by the FDA as the first molecular targeted drug for HNSCC in 2006 and had been used clinically for HNSCC in Japan since 2013. However, the effect of cetuximab is limited to a portion of patients, and other EGFR inhibitors, such as gefitinib or erlotinib, have been shown to have no benefit on HNSCC. Thus, the establishment of novel molecular targets is required for the benefit of HNSCC patients.

The MET oncogene, which is located on chromosome 7q31, encodes a receptor, tyrosine kinase, activated by binding with hepatocyte growth factor (HGF), a sole ligand of MET [5] [6]. MET activation leads to cell proliferation, migration, motility, invasion, survival and other malignant features mainly through the RAS-RAFMEK-ERK and PI3K-AKT-mTOR pathways [7] [8]. Activating germline mutations in the MET gene are detected in the majority of hereditary papillary renal cell carcinomas, whereas somatic mutations are relatively rare, except for a portion of sporadic papillary renal cell carcinomas or childhood hepatocellular carcinomas [9] [10]. On the other hand, when amplified, wild-type MET can activate the downstream pathway without HGF and promote malignant features of the cells. In fact, MET amplification is detected in various cancers, including $10 \%$ of gastric cancers, $12 \%$ of colorectal cancers and $14 \%$ of renal cancers [11]-[13]. In non-small cell lung cancer (NSCLC), while MET amplification is detected in $7 \%$ of primary NSCLC, the incidence increases to $22 \%$ in NSCLC from patients showing resistance to gefitinib, an EGFR-tyrosine kinase inhibitor (EGFR-TKI) [14]. In vitro, it has been demonstrated that NSCLC cell lines with MET amplification overcome inhibition of gefitinib by activating HER3, a member of the EGFR family of proteins, and subsequently activating the PI3K-AKTmTOR pathway [14]. MET is also reported to be associated with resistance to other EGFR-TKIs, chemotherapy and radiotherapy [15]-[17]. Thus, a number of MET inhibitors have been developed and some of them are being tested clinically [8] [18].

In HNSCC, numerous studies have demonstrated that the MET protein is overexpressed in most cases, suggesting a promotive role of MET in HNSCC oncogenesis [19]-[22]. By contrast, only two studies have reported mutations or amplification of the MET gene in HNSCC. Di Renzo et al. showed the activating MET mutations in 4 of 15 (27\%) European HNSCC [23], while Seiwert et al. showed mutation and copy gain of the MET in 9 of 66 (14\%) and 15 of 23 (65\%) HNSCC, respectively, in the USA [19]. Recently, Ando et al. reported a novel MET mutation in a maxillary carcinosarcoma, a subtype of HNSCC [24]. However, no study has been reported on the MET mutation or amplification in HNSCC of an Asian population, including Japanese people. In the present study, we examined amplification or copy number alterations of the MET gene by droplet digital PCR (ddPCR) and quantitative real time PCR (qPCR) in 54 Japanese HNSCC cases. We also analyzed mutation of the MET by Sanger sequencing. So far as we know, this is the first report of analyses of copy number and mutation of the MET gene in Japanese HNSCC. Preferential copy gain of the MET in hypopharyngeal and laryngeal cancer suggested the site-specific oncogenesis of HNSCC and could provide a possible molecular target in a subset of hypopharyngeal and laryngeal cancer. 


\section{Materials and Methods}

\subsection{Patients and Tissues}

Fifty-six patients (46 males, 8 females) were included in this study. Inclusion criteria were as follows: (1) Patients underwent surgical resection at the Department of Otolaryngology and Head and Neck Surgery of the University of Tokyo from April, 2008 to June, 2009; (2) Tumors were pathologically diagnosed as squamous cell carcinoma; (3) Tumors were enough large to get 3 to $5 \mathrm{~mm}$ cubed specimen. Patients with skin cancer and esophageal cancer were excluded. The mean age was 65.8 years old (y.o.) (from 41 y.o. to 79 y.o., median 67 y.o.). They provided informed, written consent in accordance with the ethics board of the University of Tokyo. The primary sites of HNSCC were the oral cavity, in 21 cases, the oropharynx, in 8 cases, the hypopharynx, in 12 cases and the larynx, in 13 cases (Table 1). The number of HNSCC with the clinical stages I, II, III and IV was 4, 9, 15 and 26 cases, respectively. Among them, 13 HNSCC are recurrent cases, with clinical stages III and IV in 5 and 8 cases, respectively. After surgical resection, specimens were quickly frozen in liquid nitrogen or preserved in Allprotect Tissue Reagent (Qiagen, Alameda, CA, USA) and stored at $-80^{\circ} \mathrm{C}$.

\subsection{DNA Extraction}

Genomic DNAs were extracted using the AllPrep Mini Kit (Qiagen) and the concentration of DNA was measured by Nanodrop ND-2000 spectrophotometer (Nanodrop Technologies, Rockland, DE, USA).

\subsection{Droplet Digital PCR (ddPCR)}

Fifty to $100 \mu \mathrm{g}$ of each template DNA was digested with $0.1 \mu \mathrm{l}$ of restriction enzyme MseI, in $20 \mu \mathrm{l}$ of reaction

Table 1. Clinico-pathological features of HNSCCs with and without copy gain of the MET gene.

\begin{tabular}{|c|c|c|c|c|}
\hline & \multirow{2}{*}{ Total } & \multicolumn{2}{|c|}{ Tumors with copy gain of the $M E T$} & \multirow{2}{*}{$p$ value } \\
\hline & & yes $(\%)$ & no (\%) & \\
\hline Number of patients & 54 & $10(19)$ & $44(81)$ & \\
\hline Average Age (y.o.) & 65.6 & 70.5 & 64.8 & 0.009 \\
\hline Sex & & & & NS* \\
\hline Male & 46 & $10(22)$ & $36(78)$ & \\
\hline Female & 8 & $0(0)$ & $8(100)$ & \\
\hline Primary Site & & & & 0.018 \\
\hline Oral Cavity & 21 & $1(5)$ & $20(95)$ & $<0.05$ \\
\hline Oropharynx & 8 & $0(0)$ & $8(100)$ & NS* \\
\hline Hypopharynx & 12 & $4(33)$ & $8(67)$ & NS* \\
\hline Larynx & 13 & $5(38)$ & $8(62)$ & $<0.05$ \\
\hline Clinical Stage & & & & NS* \\
\hline 1 & 4 & $0(0)$ & $4(100)$ & \\
\hline 2 & 9 & $1(11)$ & $8(89)$ & \\
\hline 3 & 15 & $2(13)$ & $13(87)$ & \\
\hline 4 & 26 & $7(27)$ & $19(73)$ & \\
\hline Smoking History & & & & NS* \\
\hline yes & 45 & $9(20)$ & $36(80)$ & \\
\hline no & 9 & $1(11)$ & 8 (89) & \\
\hline
\end{tabular}

*NS: not significant. 
mixture at $37^{\circ} \mathrm{C}$ for 2 hours. An aliquot of this reaction mixture $(5 \mu \mathrm{l})$ was mixed with Supermix for probes (Bio-Rad, Hercules, CA, USA), $0.3 \mu \mathrm{l}$ of forward and reverse primers (each $100 \mu \mathrm{M}$ ) of the MET and RPP30 genes and $0.2 \mu \mathrm{l}$ of probes (each $100 \mu \mathrm{M}$ ) of the MET and RPP30 genes for ddPCR. The RPP30 gene, encoding ribonuclease P/MRP $30 \mathrm{kDa}$ subunit, is located on chromosome 10 in two copies in a diploid genome and is used as a control of copy number analysis. A total of $20 \mu \mathrm{l}$ of the reaction mixture was loaded onto the QX200 Droplet Generator (Bio-Rad) together with $70 \mu \mathrm{l}$ of droplet-generating oil to generate about 20 thousands of droplets. The oil/reagent emulsion was transferred to a 96 well plate (Eppendorf AG, Hamburg, Germany) and the samples were amplified on the conventional Bio-Rad T100 Thermal Cycler (Bio-Rad) with PCR condition as follows; $95^{\circ} \mathrm{C}$ for $10 \mathrm{~min}$, followed by 40 cycles of $94^{\circ} \mathrm{C}$ for $30 \mathrm{sec}$ and $60^{\circ} \mathrm{C}$ for $60 \mathrm{sec}$, with a final extension at $98^{\circ} \mathrm{C}$ for $10 \mathrm{~min}$. Subsequently, the plate was loaded onto the QX200 Droplet Reader (Bio-Rad) and individual droplets were counted as positive or negative based on the presence or absence of fluorescence from the PCR products. The numbers of targeted sequences of the MET and RPP30 genes in the mixture were determined by the QuantaSoft Software (Bio-Rad). The copy number of the MET gene was calculated by comparing the number of targeted sequences of the MET gene to that of the RPP30 gene. To evaluate the MET copy number accurately, we analyzed two distinct fragments of MET: a region within exon 14 and a region spanning intron 20exon 21 of the MET gene. Primers and probes used for ddPCR are described in Table S1.

\subsection{Real-Time PCR with TaqMan Detection}

Thecopy number of the MET gene was analyzed using the Applied Biosystems 7300 Real Time PCR System (Applied Biosystems, Foster City, CA, USA). The qPCR mixture contained $10 \mu \mathrm{l}$ of $2 \times$ Real-Time PCR Kit-FP (Diatheva, Fano, Italy), $1 \mu \mathrm{l}$ of the TaqMan Copy Number Target Assay (Hs02884964_cn, spanning intron20 and exon 21 and Hs01106926_cn, within exon17; Applied Biosystems), $1 \mu \mathrm{l}$ of the TaqMan Copy Number Reference Assay (hTERT, Applied Biosystems), $4 \mu \mathrm{l}$ of nuclease-free water, and $1 \mu \mathrm{l}$ of template DNA (diluted to a concentration of $5 \mathrm{ng} / \mu \mathrm{l})$. PCR conditions were as follows; $95^{\circ} \mathrm{C}$ for $10 \mathrm{~min}$, followed by 40 cycles of $95^{\circ} \mathrm{C}$ for $15 \mathrm{sec}$ and $60^{\circ} \mathrm{C}$ for $1 \mathrm{~min}$. Each sample was run in triplicates. After amplification, the results were analyzed using the CopyCaller Software (Applied Biosystems). The software performed a comparative $\mathrm{Ct}(\Delta \Delta \mathrm{Ct}$ ) relative quantification analysis of the real time PCR product and determined the number of copies of the target sequence.

\subsection{Sequencing Analysis}

Exons 14 - 19 of the MET gene and their flanking sequences were amplified by polymerase chain reaction (PCR) using KOD polymerase (Toyobo, Osaka, Japan). Primers used for PCR are described in Table S1. The PCR mixture contained $12.5 \mu \mathrm{l}$ of $2 \times$ PCR buffer, $5 \mu \mathrm{l}$ of $2 \mathrm{mM}$ dNTPs, 0.5 unit of KOD polymerase, $1 \mu \mathrm{l}$ of forward and reverse primers (each $10 \mu \mathrm{M}$ ), 50 ng of template DNA and distilled water to make a reaction mixture of 25 $\mu$ l. PCR conditions were as follows: $94^{\circ} \mathrm{C}$ for 2 minutes, followed by 35 cycles of $98^{\circ} \mathrm{C}$ for $5 \mathrm{sec}, 57^{\circ} \mathrm{C}$ for 10 sec and $68^{\circ} \mathrm{C}$ for $10 \mathrm{sec}$, with a final extension at $68^{\circ} \mathrm{C}$ for $7 \mathrm{~min}$. PCR products were separated by electrophoresis on agarose gel, and fragments were cut from the gel and purified by Wizard SV Gel and PCR Clean-Up System (Promega, Madison, WI, USA). Nucleotide sequences were determined by direct sequencing (Fasmac, Kanagawa, Japan) and results were analyzed using the software Lasergene (Dnastar, Madison, WI, USA).

\subsection{Statistical Analysis}

Statistical analysis was done using the software Statmate. The significance level was set at $p<0.05$.

\section{Results}

\subsection{Evaluation of Copy Number of the MET Gene in HNSCC}

The copy number of the MET gene was analyzed in each HNSCC DNA both by ddPCR and by qPCR. In each method, two separated fragments of the MET gene, a fragment spanning intron 20 to exon 21 and that within exon 14 of the MET for ddPCR and a fragment spanning intron 20 to exon 21 and that within exon 17 of the MET for qPCR, were examined. The results obtained from the two fragments from exons 14 and 21 by ddPCR were very well correlated with each other, with a correlation coefficient of 0.96 (Figure 1(a)). On the other hand, the correlation coefficient of the results from two fragments from exons 17 and 21 by qPCR 


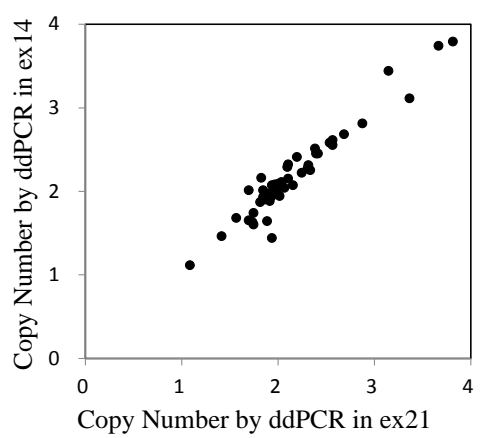

(a)

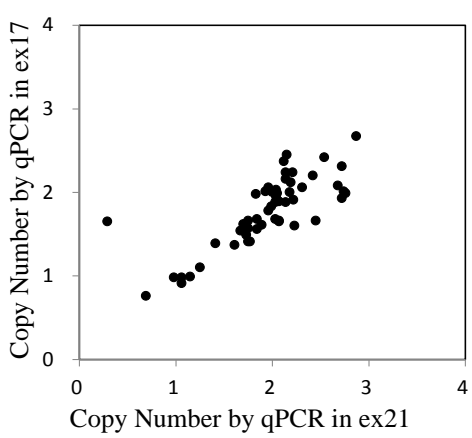

(b)

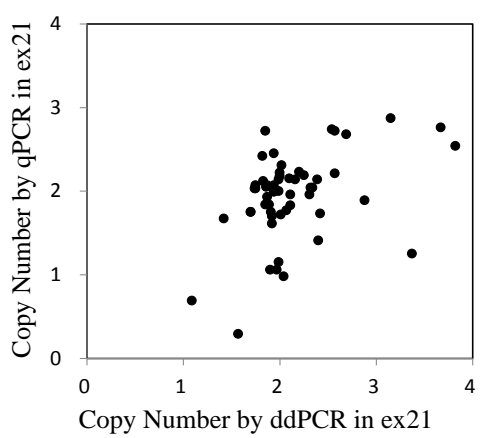

(c)

Figure 1. Copy number of the MET gene by droplet digital PCR (ddPCR) and quantitative real time PCR (qPCR). (a) Correlation between the MET copy number in exon 21 and that in exon 14 by ddPCR; (b) Correlation between the MET copy number in exon 21 and that in exon 17 by qPCR; (c) Correlation between the MET copy number in exon 21 by ddPCR and that by qPCR. Correlation coefficients are 0.96 in (a), 0.78 in (b) and 0.43 in (c).

was 0.78 (Figure 1(b)), which was significantly lower than that of ddPCR. These results suggest that ddPCR is a more reliable method for assessing DNA copy numbers than qPCR. Correspondingly, when the results of a fragment spanning intron 20 to exon 21 by ddPCR are compared with those of the same fragment by qPCR, ddPCR is more sensitive in detecting copy number gain in a subset of samples with a high copy number of the MET gene, although both results are significantly correlated with each other, with a correlation coefficient of 0.43 (Figure 1(c)). On the basis of these findings, we performed further analyses by ddPCR.

\subsection{Clinical Characteristics of HNSCC Showing Copy Gain of the MET Gene}

The results of the copy number of the MET by ddPCR in 54 Japanese HNSCC are shown in Figure 2. If we define the copy gain of MET when the results of the MET copy number in the fragments analyzed by ddPCR were over 2.5 copies, 10 of 54 (19\%) Japanese HNSCC showed copy gain of the MET gene. Gevensleben et $a l$. validated that setting the cut off value of target gene: control gene ratio at 1.25 effectively distinguished amplified samples from non-amplified samples when analyzing plasma cell-free DNA [25]. This report supports the validity of our cut off value of 2.5 copies. As summarized in Table 1, the HNSCC cases showing copy gain of $M E T$ were in significantly older people than those without the copy gain. Furthermore, the incidence of copy gain of the MET was higher in hypopharyngeal cancer (4 of 12;33\%) and in laryngeal cancer (5 of $13 ; 38 \%$ ) than in oral cancer ( 1 of $21 ; 5 \%$ ) or in oropharyngeal cancer (none of $8 ; 0 \%$ ). No significant difference in other characteristics, including sex, smoking status and clinical stages, was observed between HNSCC cases with and without copy gain of the MET gene. Moreover, as shown in Kaplan-Meier curves in Figure 3, the overall survival and the recurrence-free survival did not show any significant difference between the HNSCC cases with and without copy gain of the MET gene.

\subsection{Detection of the MET Gene Mutation in HNSCC}

Next, mutation of the MET gene was examined in the regions from exon 14 to exon 20 encoding a transmembrane domain, a juxtamembrane domain and a tyrosine kinase domain, which were the hot spots of the known activating mutations. Sanger sequencing identified base substitutions in 2 cases: one was a somatic G to A transition in exon 15 causing amino acid substitution from arginine to glutamine in codon 1040 (R1040Q) of the MET gene (Figure 4), while the other was a silent mutation in exon 16 without accompanying splicing alteration. R1040Q is a novel mutation in the juxtamembrane domain of MET protein in laryngeal cancer ofclinical stage 3.

\section{Discussion}

HNSCCs can be mainly divided into six categories on the basis of their primary sites of development, i.e. sinonasal, nasopharyngeal, oral, oropharyngeal, hypopharyngeal and laryngeal cancer. It is important to note that the etiological factors of HNSCC are different depending on their primary sites. For example, physical stimulation 


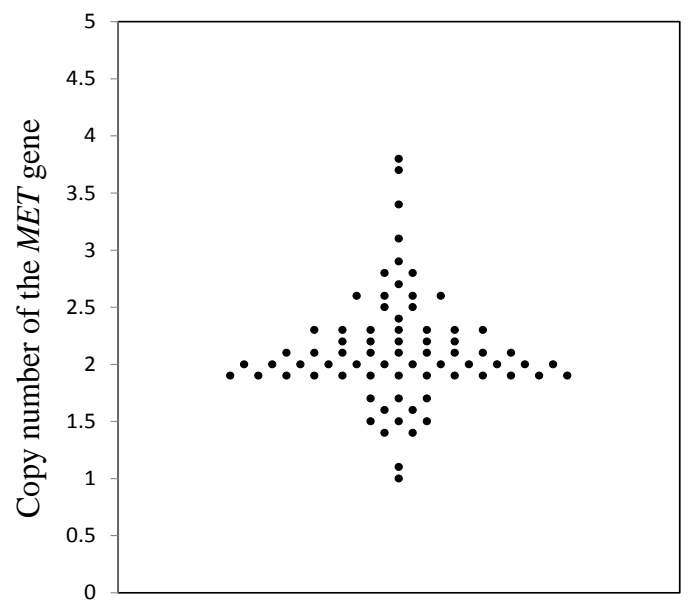

Figure 2. Copy number of the MET gene by droplet digital PCR (ddPCR).

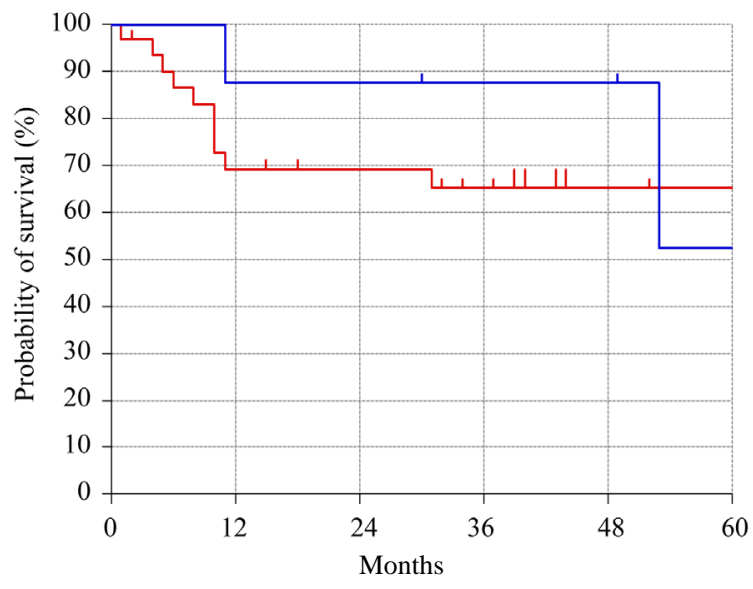

(a)

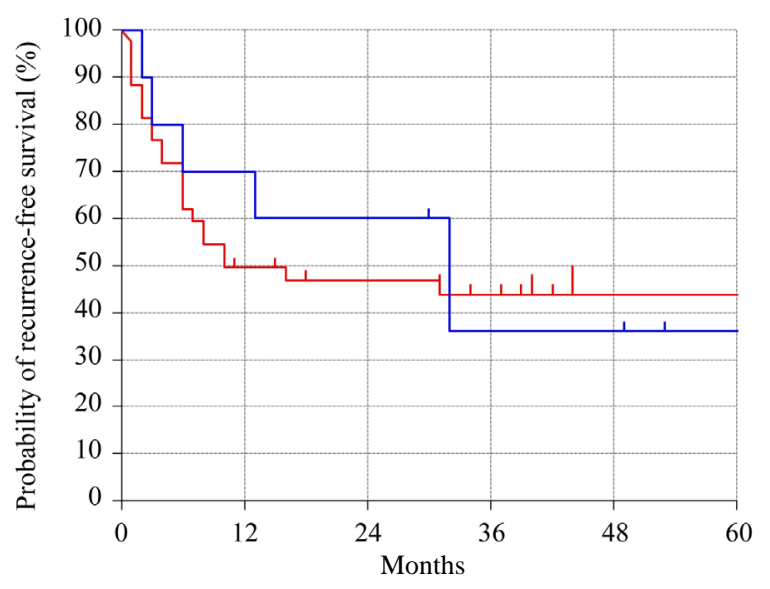

(b)

Figure 3. Kaplan-Meier plot of the overall survival (a) and recurrence-free survival (b) of the HNSCC patients with (blue) or without (red) copy number gain of the MET gene. Thirteen recurrent HNSCC cases were excluded from the analysis of the overall survival in A. (a) Overall survival in 41 cases; (b) Recurrence-free survival in 54 cases.

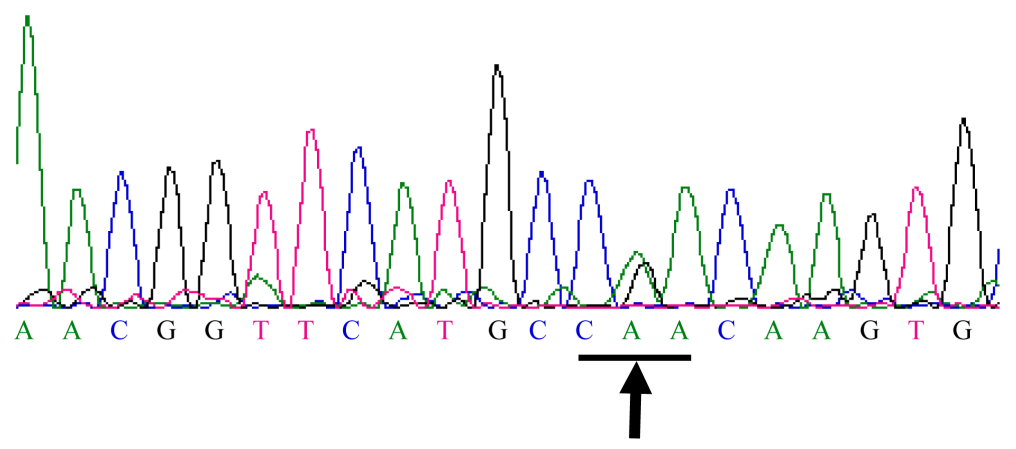

Figure 4. Sanger sequencing of the MET gene in a laryngeal cancer with a clinical stage 3, showing a missense mutation from arginine (CGA) to glutamine (CAA) in codon 1040 shown by arrow.

by teeth is a risk factor of oral cancer, and infection of human papillary viruses and EB viruses are considerable etiological factors for oropharyngeal and nasopharyngeal cancer, respectively, whereas tobacco smoking and alcohol drinking are potent etiological factors for almost all HNSCC [26]. Thus, understanding of such differ- 
ence in etiological factors and the resultant difference in molecular pathways of individual HNSCC is critical to identify the appropriate molecular targets for each HNSCC. In the present study, we demonstrated that copy gain of the MET was frequently observed in hypopharyngeal cancer (4 of 12; 33\%) and laryngeal cancer (5 of 13 ; $38 \%$ ), whereas copy gain of the MET was rarely observed in oral cancer (1 of $21 ; 5 \%)$ and not observed in oropharyngeal cancer (0 of 8; 0\%). Involvement of MET in hypopharyngeal and laryngeal cancer suggests the contribution of smoking status, although our results failed to show a significant correlation between smoking and copy gain of the MET gene. This is the first report of the primary site dependent feature of copy gain of theMET in hypopharyngeal or laryngeal cancer. No copy gain of the MET in oropharyngeal cancer suggests that oncogenesis by HPV infection does not require a MET-activating pathway, although HPV infection is not the sole etiological cause of oropharyngeal cancer. These findings provide a hypothesis that MET could serve as a possible molecular target for a subset of hypopharyngeal or laryngeal cancer, although the number of patients is not large and it is necessary to examine later whether MET copy number is an independent etiological factor.

We also found that the HNSCC cases showing the MET copy gain were in significantly older patients than those without copy gain of the MET gene (Table 1). This might be caused by unknown biological aspects related to MET activation in oncogenesis of HNSCC. Alternatively, since patients of laryngeal cancer are relatively older (average 70.6 y.o.) than those of other types of cancer (average 65.3 y.o.) in the present study, while 5 of 10 (50\%) HNSCC with copy gain of the MET, but only 8 of 44 (18\%) HNSCC without copy gain of the $M E T$, are laryngeal cancer, the average age of HNSCC with copy gain of the MET might become higher in the present analysis.

The incidence and the precise copy number of the MET in our study are much lower than those in the previous study. Seiwert et al. reported that the MET copy number was more than 10 in 3/23 (13\%) HNSCC and more than 4 in 18/23 (78\%) HNSCC when detected by qPCR using SYBR Green Probe or FISH analyses [19]. In contrast, the MET copy number in the present study was more than 2.5 in 10/54 (19\%) HNSCC and more than 3 in 4/54 (7\%) with maximum copy number of 3.81 by using ddPCR. In addition, MET copy number in the present study detected by qPCR using TaqMan Probe was less than 3 in all 54 HNSCC cases. These results suggest that the degree of copy gain of the MET in Japanese HNSCC would be lower compared to Caucasian HNSCC, although we should carefully interpret the results of the two studies obtained by different methods for estimating the MET copy number. In this connection, it is noteworthy that the incidence of copy gain of the $M E T$ in NSCLC increases from $7 \%$ to $22 \%$ in recurrent tumors after the treatment of EGFR-TKI [14] [27]. In addition, it is demonstrated that MET activation is associated with resistance to other types of EGFR-TKI, chemotherapy and radiotherapy in various cancer cells [15]-[17]. In the present study, however, copy gain of the MET was observed in only 1 of 13 (8\%) recurrent HNSCC and no significant correlation with any clinicopathological characteristics was observed (Table S2). Further analysis of the MET in a larger number of HNSCC in the Japanese population is necessary.

We have also reported 2 nucleotide substitutions of the MET gene by Sanger sequencing. One was a silent mutation and the other was a novel missense mutation, R1040Q, corresponding to the juxtamembrane domain of MET, although pathological significance of R1040Q mutation is to be elucidated by further study. The frequency of mutations with amino acid substitution was 1.8\% (1 of 54) in our study, which was lower than that in the previous studies by Di Renzo et al. (27\%) and Seiwert et al. (14\%) [19] [23].

In the current study, we have also shown predominant features of ddPCR in quantification of DNA fragments. Droplet digital PCR is categorized as the third generation PCR in comparison with qPCR, so-called the second generation PCR, due to its highly quantitative ability for evaluating the amounts of the template DNA. By using limited diluted DNA samples as templates in numerous independent reactions, a single molecule of the template DNA can be amplified to give the product, while a solution without template DNA does not give the product. Then, based on the ratios of the product positive and the product negative reactions, we could calculate the definite amount of template DNA. Moreover, by comparing the amount of standard DNA present in 2 copies in the cells, we could calculate the relative copy number of the gene of interest using less than 10 ng of DNA. This principle is clearly advantageous in quantifying the template DNA, in comparison with that in qPCR. In fact, correlation of the MET copy number of the fragments from exon 21 and exon 14 by ddPCR is much better than that from exon 21 and exon 17 by qPCR $(\mathrm{R}=0.96$ vs $\mathrm{R}=0.78)$. Even if the MET gene is truncated within the gene region, the copy number of the MET of exon 21 and that of exon 17 is expected to correlate better than those of exon 21 and exon 14. Therefore, our results strongly suggest that ddPCR is more reliable for quantifying the copy number of genes. Comparison of the MET copy number of exon 21 by ddPCR and by qPCR sug- 
gests that ddPCR is more quantitative than qPCR especially in samples showing a high copy number of the MET gene, which is also predicted by comparing the theoretical principles of qPCR and ddPCR. Furthermore, ddPCR is an easy and sensitive method to analyze the copy number of multiple samples at the same time quickly. Taken together, ddPCR could provide a suitable diagnostic method for detecting the copy number of target genes.

\section{Conclusion}

We have demonstrated that copy gain of the MET is detected in about one third of hypopharyngeal and laryngeal cancer in Japanese patients. This is the first report showing high incidence of the MET copy gain in hypopharyngeal and laryngeal cancer in comparison with oral or oropharyngeal cancer. These findings suggested that MET could provide a possible molecular target in a subset of hypopharyngeal and laryngeal cancer when copy gain of the $M E T$ was detected by ddPCR as a companion marker.

\section{Acknowledgements}

The authors thank the members of the Division of Molecular Pathology and the Department of OtolaryngologyHead and Neck Surgery for their constructive criticism and fruitful discussion. This work was supported in part by JSPS KAKENHI Grant Numbers 25290051 and 26640121 for Y.M; a Grant-in-Aid for the Third-Term Comprehensive Control Research for Cancer from the Ministry of Health, Labor, and Welfare, Japan [Y.M.].

\section{References}

[1] Jemal, A., Thun, M.J., Ries, L.G., Howe, H.L., Weir, H.K., Center, M.M., et al. (2008) Annual Report to the Nation on the Status of Cancer, 1975-2005, Featuring Trends in Lung Cancer, Tobacco Use, and Tobacco Control. Journal of the National Cancer Institute, 100, 1672-1694. http://dx.doi.org/10.1093/jnci/djn389

[2] Carvalho, A.L., Nishimoto, I.N., Califano, J.A. and Kowalski, L.P. (2005) Trends in Incidence and Prognosis for Head and Neck Cancer in the United States: A Site-Specific Analysis of the SEER Database. International Journal of Cancer, 114, 806-816. http://dx.doi.org/10.1002/ijc.20740

[3] Bonner, J.A., Harari, P.M., Giralt, J., Azarnia, N., Shin, D.M., Cohen, R.B., et al. (2006) Radiotherapy plus Cetuximab for Squamous-Cell Carcinoma of the Head and Neck. The New England Journal of Medicine, 354, 567-578 http://dx.doi.org/10.1056/NEJMoa053422

[4] Vermorken, J.B., Mesia, R., Rivera, F., Remenar, E., Kawecki, A., Rottey, S., et al. (2008) Platinum-Based Chemotherapy plus Cetuximab in Head and Neck Cancer. The New England Journal of Medicine, 359, 1116-1127. http://dx.doi.org/10.1056/NEJMoa0802656

[5] Park, M., Dean, M., Kaul, K., Braun, M.J., Gonda, M.A. and Vande Woude, G.F. (1987) Sequence of MET Protooncogene cDNA Has Features Characteristic of the Tyrosine Kinase Family of Growth-Factor Receptors. Proceedings of the National Academy of Sciences of the United States of America, 84, 6379-6383. http://dx.doi.org/10.1073/pnas.84.18.6379

[6] Bottaro, D.P., Rubin, J.S., Faletto, D.L., Chan, A.M., Kmiecik, T.E., Vande Woude, G.F. and Aaronson, S.A. (1991) Identification of the Hepatocyte Growth Factor Receptor as the c-Met Proto-Oncogene Product. Science, 251, 802-804. http://dx.doi.org/10.1126/science.1846706

[7] Peruzzi, B. and Bottaro, D.P. (2006) Targeting the c-Met Signaling Pathway in Cancer. Clinical Cancer Research, 12, 3657-3660. http://dx.doi.org/10.1158/1078-0432.CCR-06-0818

[8] Gherardi, E., Birchmeier, W., Birchmeier, C. and Vande Woude, G.F. (2012) Targeting MET in Cancer: Rationale and Progress. Nature Reviews Cancer, 12, 89-103. http://dx.doi.org/10.1038/nrc3205

[9] Schmidt, L., Duh, F.M., Chen, F., Kishida, T., Glenn, G., Choyke, P., et al. (1997) Germline and Somatic Mutations in the Tyrosine Kinase Domain of the MET Proto-Oncogene in Papillary Renal Carcinomas. Nature Genetics, 16, 68-73. http://dx.doi.org/10.1038/ng0597-68

[10] Park, W.S., Dong, S.M., Kim, S.Y., Na, E.Y., Shin, M.S., Pi, J.H., et al. (1999) Somatic Mutations in the Kinase Domain of the Met/Hepatocyte Growth Factor Receptor Gene in Childhood Hepatocellular Carcinomas. Cancer Research, 59, 307-310.

[11] Nakajima, M., Sawada, H., Yamada, Y., Watanabe, A., Tatsumi, M., Yamashita, J., et al. (1999) The Prognostic Significance of Amplification and Overexpression of c-met and c-erb B-2 in Human Gastric Carcinomas. Cancer, 85, 18941902. http://dx.doi.org/10.1002/(SICI)1097-0142(19990501)85:9<1894::AID-CNCR3>3.0.CO;2-J

[12] Umeki, K., Shiota, G. and Kawasaki, H. (1999) Clinical Significance of c-met Oncogene Alterations in Human Colorectal Cancer. Oncology, 56, 314-321. http://dx.doi.org/10.1159/000011985 
[13] Jardim, D.L.F., Tang, C., Gagliato, D.D.M., Falchook, G.S., Hess, K., Janku, F., et al (2014) Analysis of 1,115 Patients Tested for MET Amplification and Therapy Response in the MD Anderson Phase I Clinic. Clinical Cancer Research, 20, 6336-6345. http://dx.doi.org/10.1158/1078-0432.CCR-14-1293

[14] Engelman, J.A., Zejnullahu, K., Mitsudomi, T., Song, Y., Hyland, C., Park, J.O., et al. (2007) MET Amplification Leads to Gefitinib Resistance in Lung Cancer by Activating ERBB3 Signaling. Science, 316, 1039-1043. http://dx.doi.org/10.1126/science.1141478

[15] Cepero, V., Sierra, J.R., Corso, S., Ghiso, E., Casorzo, L., Perera, T., et al. (2010) MET and KRAS Gene Amplification Mediates Acquired Resistance to MET Tyrosine Kinase Inhibitors. Cancer Research, 70, 7580-7590. http://dx.doi.org/10.1158/0008-5472.CAN-10-0436

[16] Bowers, D.C., Fan, S., Walter, K.A., Abounader, R., Williams, J.A., Rosen, E.M. and Laterra, J. (2000) Scatter Factor/Hepatocyte Growth Factor Protects against Cytotoxic Death in Human Glioblastoma via Phosphatidylinositol 3-Kinase- and AKT-Dependent Pathways. Cancer Research, 60, 4277-4283.

[17] De Bacco, F., Luraghi, P., Medico, E., Reato, G., Girolami, F., Perera, T., et al. (2011) Induction of MET by Ionizing Radiation and Its Role in Radioresistance and Invasive Growth of Cancer. Journal of the National Cancer Institute, 103, 645-661. http://dx.doi.org/10.1093/jnci/djr093

[18] Blumenschein, G.R., Mills, G.B. and Gonzalez-Angulo, A.M. (2012) Targeting the Hepatocyte Growth Factor-cMET Axis in Cancer Therapy. Journal of Clinical Oncology, 30, 3287-3296. http://dx.doi.org/10.1200/JCO.2011.40.3774

[19] Seiwert, T.Y., Jagadeeswaran, R., Faoro, L., Janamanchi, V., Nallasura, V., Dinali, M.E., et al. (2009)The MET Receptor Tyrosine Kinase Is a Potential Novel Therapeutic Target for Head and Neck Squamous Cell Carcinoma. Cancer Research, 69, 3021-3031. http://dx.doi.org/10.1158/0008-5472.CAN-08-2881

[20] Christensen, J.G., Burrows, J. and Salgia, R. (2005) c-Met as a Target for Human Cancer and Characterization of Inhibitors for Therapeutic Intervention. Cancer Letters, 225, 1-26. http://dx.doi.org/10.1016/j.canlet.2004.09.044

[21] Kim, C.H., Kim, C.H., Moon, S.K., Bae, J.H., Lee, J.H., Han, J.H., Kim, K. and Choi, E.C. (2006) Expression of Hepatocyte Growth Factor and c-met in Hypopharyngeal Squamous Cell Carcinoma. Acta Oto-Laryngologica, 126, 88-94. http://dx.doi.org/10.1080/00016480510037014

[22] Murai, M., Shen, X., Huang, L., Carpenter, W.M., Lin, C.S. and Silverman, S. (2004) Overexpression of c-met in Oral SCC Promotes Hepatocyte Growth Factor-Induced Disruption of Cadherin Junctions and Invasion. International Journal of Oncology, 25, 831-840.

[23] Di Renzo, M.F., Olivero, M., Martone, T., Maffe, A., Maggiora, P., Stefani, A.D., et al. (2000) Somatic Mutations of the MET Oncogene Are Selected during Metastatic Spread of Human HNSC Carcinomas. Oncogene, 19, 1547-1555. http://dx.doi.org/10.1038/sj.onc.1203455

[24] Ando, M., Saito, Y., Morikawa, T., Omura, G., Kobayashi, K., Akashi, K., et al. (2015) Maxillary Carcinosarcoma: Identification of a Novel MET Mutation in both Carcinomatous and Sarcomatous Components through Next Generation Sequencing. Head \& Neck, 37, E179-E185. http://dx.doi.org/10.1002/hed.24043

[25] Gevensleben, H., Garcia-Murillas, I., Graeser, M.K., Schiavon, G., Osin, P., Parton, M., Smith, I.E., Ashworth, A. and Turner, N.C. (2013) Noninvasive Detection of HER2 Amplification with Plasma DNA Digital PCR. Clinical Cancer Research, 19, 3276-3284. http://dx.doi.org/10.1158/1078-0432.CCR-12-3768

[26] Chung, C.H., Parker, J.S., Karaca, G., Wu, J., Funkhouser, W.K., Moore, D., et al. (2004) Molecular Classification of Head and Neck Squamous Cell Carcinomas Using Patterns of Gene Expression. Cancer Cell, 5, 489-500. http://dx.doi.org/10.1016/S1535-6108(04)00112-6

[27] Cappuzzo, F., Jänne, P.A., Skokan, M., Finocchiaro, G., Rossi, E., Ligorio, C., et al. (2009) MET Increased Gene Copy Number and Primary Resistance to Gefitinib Therapy in Non-Small-Cell Lung Cancer Patients. Annals of Oncology, 20, 298-304. http://dx.doi.org/10.1093/annonc/mdn635 


\section{Supplement}

Table S1. Primers and probes used for droplet digital PCR and Sanger sequencing. (a) Droplet digital PCR; (b) Sanger sequencing of exons and their flanking sequences.

(a)

\begin{tabular}{ll}
\hline Exon $14\left(5^{\prime}-3^{\prime}\right)$ & \\
\hline Sense & TTAGTTCGCTACGATGCAAGAG \\
Probe & FAM-CACACTCCTCATTTGGATAGGCTTG-IBFQ \\
Anti-Sense & GGCTTACACTTCGGGCACT \\
\hline & \\
\hline Exon 14 (5' - 3') & \\
\hline Sense & TTAGTTCGCTACGATGCAAGAG \\
Probe & FAM-CACACTCCTCATTTGGATAGGCTTG-IBFQ \\
Anti-Sense & GGCTTACACTTCGGGCACT \\
\hline
\end{tabular}

(b)

\begin{tabular}{lll}
\hline Exon & Sense $\left(5^{\prime}-3^{\prime}\right)$ & Anti-Sense $\left(5^{\prime}-3^{\prime}\right)$ \\
\hline Exon14 & TCATTTTAGTGGAAGCAAGCA & GTTTGGGCTTCAACAGGTAAAA \\
Exon15 & GATTTTGTGAGACGAGGCAAT & CTGGCCCTGAGTTTTAGAAGAA \\
Exon16 & ATTTGATGAGGCCGATGTTACT & GCCAACATGCTTCCAATAGTTT \\
Exon17 & TTGTTGGAAGTCTGACCATGAG & GGAACGCATCTGGAGTTAATTT \\
Exon18 & GACTCCTAAGCAGCTGGGACTA & ATTCTCCCTCCCCAACACTAAT \\
Exon19 & TAGCAACCAAAGTAAACCCACA & TCTCATCTCTTCCCTTCTGAGC \\
\hline
\end{tabular}

Table S2. Clinico-pathological features of recurrent HNSCCs with and without copy gain of the MET gene.

\begin{tabular}{|c|c|c|c|c|}
\hline & \multirow{2}{*}{ Total } & \multicolumn{2}{|c|}{ Tumors with copy gain of the $M E T$} & \multirow{2}{*}{$p$ value } \\
\hline & & yes $(\%)$ & no (\%) & \\
\hline Number of patients & 13 & $1(8)$ & $12(92)$ & \\
\hline Average Age (y.o.) & 67.3 & 70.0 & 67.1 & NS \\
\hline Sex & & & & NS \\
\hline Male & 11 & $1(9)$ & $10(91)$ & \\
\hline Female & 2 & $0(0)$ & $2(100)$ & \\
\hline Primary Site & & & & NS \\
\hline Oral Cavity & 5 & $0(0)$ & $5(100)$ & \\
\hline Oropharynx & 2 & $0(0)$ & $2(100)$ & \\
\hline Hypopharynx & 2 & $0(0)$ & $2(100)$ & \\
\hline Larynx & 4 & $1(25)$ & $3(75)$ & \\
\hline Clinical Stage & & & & NS \\
\hline 1 & 0 & $0(0)$ & $0(0)$ & \\
\hline 2 & 0 & $0(0)$ & $0(0)$ & \\
\hline 3 & 5 & $0(0)$ & $5(100)$ & \\
\hline 4 & 8 & $1(12)$ & $19(87)$ & \\
\hline Smoking History & & & & NS \\
\hline yes & 12 & $1(8)$ & $11(92)$ & \\
\hline no & 1 & $0(0)$ & $1(100)$ & \\
\hline
\end{tabular}

*NS: not significant. 